\title{
(Neo-)Marxistische Politische Ökonomie als Gegenprogramm zur Standardökonomik: Replik zum Kommentar von Frank Beckenbach
}

\author{
Hardy Hanappi
}

Online publiziert: 27. Februar 2019

(C) List-Gesellschaft e.V. 2019

Der Kommentar von Frank Beckenbach stellt einen eigenständigen Text zum Thema des Kapitels dar. Schon in den ersten Absätzen seines Kommentars stellt FB klar, dass er meine „Überlegungen“ zwar für interessant aber als „ungeeignet“ für die Themenstellung hält. Auch wenn das auf einer nicht weiter begründeten pauschalen Ablehnung der Struktur des Kapitels zu beruhen scheint, so dürfte FB doch aufgefallen sein, dass die Präsentation eines parallelen Textes zur selben Kapitelüberschrift den Charakter eines Kommentars verliert. Es findet sich daher auch die etwas versöhnlichere Formulierung, dass er nur ,die Besonderheit des theoriegeschichtlichen Ausgangspunktes ... von Marx schärfen ... und diskutieren (wolle, HH)““. Da die inhaltliche Kritik am Kapitel weitestgehend im Dunkel bleibt, will ich im Folgenden von der konstruktiveren Annahme ausgehen, dass FB seinen Text als dazu komplementäre Ergänzung mit ihm wichtig scheinenden Aspekten versteht. Dabei können einige übereinstimmende und einige divergierende Standpunkte klarer dargestellt werden.

Die wichtigsten gemeinsam betonten Eigenschaften der neo-marxistischen Ansätze sind rasch aufgezählt:

1. Die Berufung auf das Werk von Karl Marx ist auch deshalb schwierig, weil es fragmentarisch und in seiner Zusammensetzung unsystematisch vorliegt.

2. Der Begriff des sozialen Werts und die Entwicklung der Formen in denen er historisch erscheint steht im Zentrum der Marxschen Analyse.

This reply refers to the commentary article available online at https://doi.org/10.1007/s41025-01900146-3.

\footnotetext{
H. Hanappi $(\bowtie)$

Technische Universität Wien, Wien, Österreich

E-Mail: hanappi@gmail.com
} 
3. Durch die Verschmelzung der Wertanalyse mit Theoriefragmenten zur Klassendynamik und zum technischen Fortschritt gelingt es Marx eine umfassendere Gesellschaftsdynamik zu beschreiben als dies neoklassische Ansätze auf Grund ihrer Axiomatik bieten können. Schumpeter folgend nennt FB das Entwicklungstheorie.

4. Diese Gesellschaftsdynamik eröffnet bei Marx - und in der Folge auch in neomarxistischen Ansäzen - die Möglichkeit einer stärkeren Gliederung der beobachteten historischen Entwicklung. Es können längerfristig gültige Verhältnisse von spezifischen, kürzerfristigen Ausprägungen derselben unterschieden werden.

Im Sinne des methodischen Vorrangs der Behandlung von Widersprüchen muss aber nun auch auf die Unterschiedlichkeiten zwischen dem Paralleltext von FB und dem Kapitel eingegangen werden.

1. Eine in der marxistischen Literatur altbekannte Auseinandersetzung betrifft die Existenz eines Bruches im Schaffen von Marx. Die von Marx vor diesem Bruch (berühmt ist Louis Althussers ausführliche Behandlung dieser ,rupture“) verfassten Schriften seien streng von seinen späteren, wissenschaftlicheren Werken zu unterscheiden. Dementsprechend pocht FB auf den ,„ökonomischen Kern“, der sich nur im Spätwerk von Marx finde. Demgegenüber wird in meinem Kapitel von einem durchgehenden Lebenswerk von Marx ausgegangen, eine Abwertung der frühen Schriften scheint mir dem Verständnis seiner Intentionen abträglich zu sein.

2. Darauf aufbauend wird in meinem Kapitel auch weniger Marx Exegese zur Auffindung des wahren Marx in einzelnen ökonomischen Passagen betrieben. Stattdessen wird versucht den lebenslang durchgehenden methodischen Ansatz zu identifizieren, wobei klarerweise dem Hegelschen Erbe und dem Begriff der Entfremdung größerer Stellenwert zukommt. Deshalb ist auch die Entwicklung meiner vier Typen von Neo-Marxismus mit unterschiedlichen Generationen von Marx Rezeptionen verknüpft, die eine unterschiedliche Entfernung vom Originaltext spiegeln. Dabei ist keiner dieser Typen wahrer als der andere, weil er eine bessere Exegese als Grundlage hat. Jeder trägt zur Marxschen Intention bei indem er spezifische Widersprüchlichkeiten in den Griff zu bekommen versucht.

3. Im Kapitel wird die Kritik der neoklassischen Theorie wenig ausführlich behandelt, weil ja die positive Ausprägung neo-marxistischer Ansätze herausgearbeitet werden soll. Dass die Leser des Buches wissen worin die Kernannahmen der Neoklassik (sofern man diese heute überhaupt noch als einheitliche Schule identifizieren kann) bestehen wird vorausgesetzt. FB hingegen geht auf einige spezifische Anliegen von Marx ein und zeigt wie diese im Widerspruch zur Neoklassik, salopp als Standardökonomie bezeichnet, stehen. Insofern stellt sein Text zwar eine interessante komplementäre Ergänzung zum Kapitel dar, das Bindeglied zwischen spezifischen Marxschen Textstellen, das einen gemeinsamen Begriff des Neo-Marxismus rechtfertigen könnte bleibt aber im Dunkeln.

4. Bei dieser Auseinandersetzung mit der Neoklassik verheddert sich FB zeitweise in deren ideologischen Fallstricken. So konstatiert er etwa die fehlende ,akteurstheoretische Fundierung “ bei Marx (Fußnote 3); sprich: die sattsam bekannte 
Forderung der Lösung der Mikrofundierung der Makroökonomie, also des ökonomischen Ausdrucks für methodologischen Individualismus. Dementsprechend wird der Neoklassik vorgeworfen sie harmonisiere indem sie den präferenzgeleiteten Konsum einzelner Individuen in den Mittelpunkt stelle. Der Skandal ist jedoch nicht das Harmonisieren, sondern die Ignoranz gegenüber der Existenz von Klassen.

5. Eine spezielle Differenz betrifft die Einschätzung der Arbeitswerttheorie. Zwar betrachtet auch FB die neoricardianische „Widerlegung“ der Arbeitswerttheorie (Ian Steedman) offensichtlich skeptisch, er enthält sich aber weiterer Aussagen. Im Gegensatz dazu wird im Kapitel selbst angedeutet (und das nicht tiefergehend auszuführen ist sicher ein Manko des Kapitels), dass die Entwicklung einer umfassenderen Arbeitswerttheorie ein vielversprechendes Forschungsprojekt sein kann. Das wird anhand einer skizzierten IO-Darstellung des Feudalismus skizziert, die zeigen soll wie die Dynamik vielfältiger Machtausübungsformen zur Veränderung von Ausbeutungsverhältnissen führen kann, die dann mit Hilfe dieser Darstellungsform in Zeiteinheiten gemessen werden kann. Typische Mysterien, wie etwa Entstehung und Einfluss technischen Fortschritts oder der Klassendynamik, müssten dazu aufgelöst und eingebaut werden. Dass aber letztlich ,alle Ökonomie auf Ökonomie der Zeit" umzulegen wäre, scheint mir nach wie vor ein interessanter Forschungsgegenstand zu sein.

6. Marktprozesse und das Gleichgewichtskonzept spielen in der Neoklassik eine tragende Rolle und FB geht dementsprechend etwas expliziter auf sie ein. Interessanterweise beschreibt FB die Vorgehensweise von Marx analog zum Vorgehen der neueren empirisch orientierten I-O Analyse, die von ihm an anderer Stelle geschmäht wird. Mein eigener Standpunkt dazu ist ähnlich: Die Auffassung von Markt als einer Ausprägung eines Gleichgewichtszustands ist zu ersetzen durch die Beschreibung von diversen Marktmechanismen, die in aller Regel Ungleichgewichtszustände perpetuieren. Das Gleichgewichtskonzept bleibt bei FB auch insofern etwas unterbeleuchtet als seine Wirkung zur Sterilisierung dynamischer Prozesse im Rahmen theoretischer Modellbildung nur tangiert wird. Die Bedeutung von Ungleichgewichtsmodellen als wichtiger Komponente neo-marxistischer Modelle, also der Gegensatz zur Stabilitätsanalyse der Neoklassik, gerät dadurch leider etwas aus dem Blickfeld.

7. Als wichtigste Anforderungen für die „Modellierung des Marxschen Konzepts“ zählt FB schließlich vier Punkte auf. Grob gesprochen beziehen die Forderungen sich auf: I. Tiefgehende sektorale Gliederung mit adäquater Geld- und Preistheorie, II. Adäquate Verteilungstheorie, III. Darauf basierende Theorie technischen Fortschritts und Arbeitsmarkttheorie, IV. Adäquate Entwicklungstheorie für längerfristige Reproduktion mittels spezifischen Marktprozessen.

Schon die Aufzählung macht klar, dass diese Übersetzung der von FB herausgearbeiteten Marxschen Kernanliegen den Stempel neoklassischer Begriffskonstrukte trägt. Wenig überraschend stellt er denn auch fest, dass keiner der von mir en passant verwendeten Modellansätze allen von ihm zusammengestellten vier Punkten Rechnung trägt. Anders als von FB suggeriert sollte auch keiner dieser Modellansätze ein „Marxsches Kernmodell“" präsentieren, schon gar nicht eines, das den von FB erstellten Forderungskatalog erfüllt. Als Detail am Rande sei vermerkt, 
dass die vielen auf Goodwin (1973) folgenden Erweiterungen dieses Modells sehr wohl Geld als auch sektorale Gliederung und technischen Fortschritt in das Modell einzubauen versuchten.

8. Schließlich ist in Bezug auf den letzten Absatz des Kommentars von FB festzuhalten, dass das Kapitel gar nicht versucht ein „Marxsches Kernmodell“, das „Marxsche Konzept ... aus der Sicht spezieller Modellierungsvarianten“ (FB) zu beurteilen. Schlicht und einfach schon deshalb nicht, weil im Gegensatz zu Beckenbachs Text gar kein wahres ökonomisches Kernmodell von Marx herausdestilliert wurde, beziehungsweise hineininterpretiert wurde. Die vorgestellten Modellvarianten dienen der Illustration einiger wichtiger theoretischer Elemente bekannter, sich neomarxistisch nennender ökonomischer Forschungsgemeinschaften, nicht mehr und nicht weniger. Der Vorwurf der „Unangemessenheit“ des Kapiteltextes geht folglich komplett in die Leere. Was FB vorschwebt ist offensichtlich seine eigene Interpretation als Grundlage für ein den angeführten Beispielen überlegenes Modell entgegenzuhalten. Diesen eine bessere Interpretation von Marx hinzuzufügen schiene ihm ,,angemessen“. Aus meiner Sicht ein Mißverständnis.

Frank Beckmann hat ein interessantes, kurzes Paper mit dem Namen „De-konstruktive Rückbesinnung auf die Marxsche Kritik der Politischen Ökonomie“ geschrieben. In seiner Struktur folgt es seiner Auffassung davon was die fünf wesentlichsten Aspekte von Marx' ökonomischer Theorie sind. Jedem dieser Aspekte fügt er eine Einschätzung darüber hinzu was die Standardökonomie dazu zu sagen hat. Eingestreut in seinen Text finden sich Bemerkungen zum Kapitel dessen Kommentar der Text eigentlich sein sollte. Wie eingangs erwähnt, fasse ich seine Arbeit als komplementäre - manchmal aber irreführende - Ergänzung zum Kapitel auf. Die besprochenen Punkte zeigen, so hoffe ich, dass die Produktivität des Widerspruchs in Marxscher Tradition in ungebrochener Vitalität am Leben ist. In diesem Sinne ist Frank Beckenbach zu danken. 